\title{
Multiplication Operators on Weighted Nakano (sss)
}

\author{
Awad A. Bakery $\mathbb{D}^{1,2}$ Afaf R. Abou Elmatty $\mathbb{D}^{2}{ }^{2}$ and OM Kalthum S. K. Mohamed $\mathbb{D}^{1,3}$ \\ ${ }^{1}$ University of Jeddah, College of Science and Arts at Khulis, Department of Mathematics, Jeddah, Saudi Arabia \\ ${ }^{2}$ Department of Mathematics, Faculty of Science, Ain Shams University, P.O. Box 1156, Cairo 11566, Abbassia, Egypt \\ ${ }^{3}$ Academy of Engineering and Medical Sciences, Department of Mathematics, Khartoum, Sudan
}

Correspondence should be addressed to OM Kalthum S. K. Mohamed; om_kalsoom2020@yahoo.com

Received 19 July 2020; Accepted 15 September 2020; Published 30 September 2020

Academic Editor: Kit C. Chan

Copyright (C) 2020 Awad A. Bakery et al. This is an open access article distributed under the Creative Commons Attribution License, which permits unrestricted use, distribution, and reproduction in any medium, provided the original work is properly cited.

In this article, we investigate the sufficient conditions on weighted Nakano sequence space to be premodular Banach (sss). We examine some topological and geometrical structures of the multiplication operators defined on weighted Nakano prequasinormed (sss).

\section{Introduction}

Summability is very important in mathematical models and has numerous implementations, such as normal series theory, approximation theory, ideal transformations, and fixed point theory. For more details, see [1-4]. By $\mathbb{C}^{\mathbb{N}}, c, \ell_{\infty}$, $\ell^{\mathrm{r}}$, and $c_{o}$, we signify the spaces of each, convergent, bounded, r-absolutely summable, and convergent to zero sequences of complex numbers. $\mathbb{N}$ indicates the set of nonnegative integers. Let $p=\left(p_{n}\right)$ and $\left(\beta_{n}\right)_{n \in \mathbb{N}}$ be sequences of positive reals, and the weighted Nakano sequence space [5] is defined by

$$
\ell_{\beta}^{\left(p_{n}\right)}=\left\{x=\left(x_{k}\right) \in \mathbb{C}^{\mathbb{N}}: \sigma(\lambda x)<\infty, \quad \text { for some } \lambda>0\right\},
$$

where $\sigma(x)=\sum_{k=0}^{\infty} \beta_{k}\left|x_{k}\right|^{p_{k}}$. And $\left(\ell_{\beta}^{\left(p_{n}\right)},\|\cdot\|\right)$ is a Banach space, where $\|x\|=\inf \{\eta>0: \sigma(x / \eta) \leq 1\}$.

When $\left(p_{n}\right) \in \ell_{\infty}$, we have $\ell_{\beta}^{\left(p_{n}\right)}=\left\{x=\left(x_{k}\right) \in \mathbb{C}^{\mathbb{N}}: \sigma(\lambda x)<\infty, \quad\right.$ for any $\left.\lambda>0\right\}$.

(1) If $\beta_{n}=1$, for all $n \in \mathbb{N}$, then $\ell_{\beta}^{\left(p_{n}\right)}$ will reduce to $\ell^{\left(p_{n}\right)}$ (see $[6,7])$

(2) If $\beta_{n}=\left(1 / p_{n}\right)$, for all $n \in \mathbb{N}$, then $\ell_{\beta}^{\left(p_{n}\right)}$ will reduce to $\ell\left(p_{n}\right)$ which is studied by many authors (see [8-10])

By $B(W, Z)$, we will indicate the set of every operators which are linear and bounded between Banach spaces $W$ and $Z$, and if $W=Z$, we write $B(W)$. The multiplication operators have a wide field of mathematics in functional analysis, for instance, in eigenvalue distributions theorem, geometric structure of Banach spaces, and theory of fixed point. For more details, see [11-16]. The s-numbers [17] have many examples such as the rth approximation number, denoted by $\alpha_{r}(V)$, is defined by $\alpha_{r}(V)=$ inf $\{\|\mathrm{V}-\mathrm{B}\|: B \in B(W, Z)$ and $\operatorname{rank}(\mathrm{B}) \leq r\}$, and the rth Kolmogorov number, denoted by $d_{r}(V)$, is defined by 
$d_{r}(V)=\inf _{\mathrm{dim} \mathrm{w}} \leq \mathrm{r}$ sup $\|\omega\|_{\leq 1}$ inf $v \in \mathrm{W}\|V \omega-\nu\|$. The following notations will be used in the sequel:

$$
\begin{gathered}
X^{s}=\left\{X^{s}(W, Z)\right\}, \quad \text { where } X^{s}(W, Z)=\left\{V \in B(W, Z)\left(S_{j}(V)\right)_{j=0}^{\infty} \in X\right\}, \\
X^{\text {app }}=\left\{X^{\mathrm{app}}(W, Z)\right\}, \quad \text { where } X^{\mathrm{app}}(W, Z)=\left\{V \in B(W, Z)\left(\alpha_{j}(V)\right)_{j=0}^{\infty} \in X\right\}, \\
X^{\mathrm{Kol}}=\left\{X^{\mathrm{Kol}}(W, Z)\right\}, \quad \text { where } X^{\mathrm{Kol}}(W, Z)=\left\{V \in B(W, Z)\left(d_{j}(V)\right)_{J=0}^{\infty} \in X\right\} .
\end{gathered}
$$

A few of operator ideals in the class of Hilbert spaces or Banach spaces are defined by distinct scalar sequence spaces, such as the ideal of compact operators $B_{c}$ formed by $\left(d_{r}(V)\right)$ and $c_{0}$. Pietsch [4] studied the quasi-ideals $\left(\ell^{r}\right)^{\text {app }}$ for $r \in(0, \infty)$, the ideals of Hilbert Schmidt operators between Hilbert spaces constructed by $\ell^{2}$, and the ideals of nuclear operators generated by $\ell^{1}$. He explained that $\bar{F}=$ $\left(\ell^{r}\right)^{\text {app }}$ for $r \in[1, \infty)$, where $\bar{F}$ is the closed class of all finite rank operators, and the class $\left(\ell^{r}\right)^{\text {app }}$ became simple Banach and small [1]. The strictly inclusion $\left(\ell^{j}\right)^{\text {app }}(W, Z)_{\neq}^{c} B(W$, $Z$ ), whenever $j>r>0, W$ and $Z$ are infinite-dimensional Banach spaces investigated through Makarov and Faried [2]. Faried and Bakery [18] gave a generalization of the class of quasi-operator ideal which is the prequasi-operator ideal, and they examined several geometric and topological structure of $\left(\ell_{M}\right)^{S}$ and $(\operatorname{ces}(r))^{S}$. On sequence spaces, Mursaleen and Noman $[19,20]$ investigated the compact operators on some difference sequence spaces. The multiplication operators on $(\operatorname{ces}(r),\|\|$.$) with the Luxemburg$ norm \|. || elaborated by Komal et al. [21] and Bakery and Abou Elmatty [5] gave the sufficient (not necessary) conditions on $\ell_{\beta}^{\left(p_{n}\right)}$ such that $S_{\ell^{\left(p_{n}\right)}}$ constructed a simple Banach prequi operator ideal. The prequasi-operator ideal $S_{\ell^{\left(p_{n}\right)}}^{\text {app }}$ became small and strictly contained for different weights and powers. The aim of this article to explain some results of $\left(\ell_{\beta}^{\left(p_{n}\right)}\right)_{\tau}$ equipped with the prequasi-norm $\tau$. Firstly, we give the sufficient conditions on weighted Nakano sequence space to form premodular Banach (sss). Secondly, we give the necessity and sufficient conditions on weighted Nakano sequence space equipped with the prequasi-norm such that the multiplication operator defined on it is bounded, approximable, invertible, Fredholm, and closed range operator.

\section{Preliminaries and Definitions}

Definition 1 (see [4]). An operator $V \in B(W)$ is called approximable if there are $\operatorname{Dr} \in F(W)$, for every $r \in \mathbb{N}$ and $\operatorname{limr} \longrightarrow \infty\|\mathrm{V}-\mathrm{Dr}\|=0$.

By $\Upsilon(W, Z)$ and $B_{c}(W, Z)$, we will indicate the space of all approximable and compact operators from $W$ to $Z$, respectively.

Theorem 1 (see [4]). If $W$ is Banach space with dim $(W)=\infty$, then $F(W)_{\ddagger}^{\subset} \Upsilon(W)_{\ddagger}^{\subset} B_{c}(W)_{\ddagger}^{c} B(W)$.
Definition 2 (see [22]). An operator $\mathrm{V} \in B(W)$ is called Fredholm if $\operatorname{dim}(\mathrm{R}(\mathrm{V})) c<\infty$, $\operatorname{dim}(\operatorname{kerV})<\infty$, and $\mathrm{R}(\mathrm{V})$ is closed, where $(\mathrm{R}(\mathrm{V})) \mathrm{c}$ denotes the complement of range $\mathrm{V}$.

The sequence $e_{\mathrm{j}}=(0,0,1,0,0, \ldots)$ with 1 in the $\mathrm{jth}$ coordinate, for every $j \in \mathbb{N}$, will be used in the sequel.

Definition 3 (see [3]). The space of linear sequence spaces $\mathrm{Y}$ is called (sss)

(1) If $e_{\mathrm{r}} \in \mathrm{Y}$ with $r \in \mathbb{N}$,

(2) Let $u=\left(u_{\mathrm{r}}\right) \in \mathbb{C}^{\mathbb{N}}, v=\left(v_{\mathrm{r}}\right) \in \mathrm{Y}$, and $\left|u_{\mathrm{r}}\right| \leq\left|v_{\mathrm{r}}\right|$, for every $r$ $\in \mathbb{N}$, then $u \in Y$. This means Y be solid,"

(3) If $\left(u_{\mathrm{r}}\right)\left(u_{r}\right)_{r=0}^{\infty} \in Y$, then $\left(u_{r / 2}\right)_{r=0}^{\infty} \in \mathrm{Y}$, wherever $(r / 2)$ means the integral part of $(r / 2)$

Definition 4 (see [23]). A subspace of the (sss) $Y_{\tau}$ is called a premodular (sss) if there is a function: $Y \longrightarrow[0, \infty)$ verifying the conditions:

(i) $\tau(y) \geq 0$, for each $y \in \mathrm{Y}$ and $\tau(y)=0 \Leftrightarrow y=\theta$, where $\theta$ is the zero element of $\mathrm{Y}$

(ii) There exists $a \geq 1$ such that $\tau(\eta y) \leq a|\eta| \tau(y)$, for all $y \in \mathrm{Y}$, and $\eta \in \mathbb{C}$

(iii) For some $b \geq 1, \tau(y+z) \leq b(\tau(\mathrm{y})+\tau(\mathrm{z}))$, for every $y$, $z \in Y$

(iv) $|\mathrm{yr}| \leq|\mathrm{zr}|$ with $r \in \mathbb{N}$, implies $\tau((\mathrm{yr})) \leq \tau((\mathrm{zr}))$

(v) For some $b 0 \geq 1, \tau((\mathrm{yr})) \leq \tau\left(\left(y_{(r / 2)}\right)\right) \leq b 0 \tau$ ((yr))

(vi) If $y=\left(y_{r}\right)_{r=0}^{\infty} \in \mathrm{Y}$, and $d>0$, then there is $\mathrm{r} 0 \in \mathbb{N}$ with $\tau\left(\left(y_{r}\right)_{r=0}^{\infty}\right)<d$

(vii) There is $t>0$ with $\tau(\nu, 0,0,0, \ldots) \geq t|\nu| \tau(1,0,0,0$, ...), for any $? \in \mathbb{C}$

Definition 5 (see [23]). The (sss) $Y_{\tau}$ is called prequasinormed (sss) if $\tau$ satisfies the Parts (i)-(iii) of Definition 4, and when the space $Y$ is complete under $\tau$, then $Y_{\tau}$ is called a prequasi-Banach (sss).

Theorem 2 (see [23]). A prequasi-norm (sss) $Y_{\tau}$, whenever it is premodular (sss).

The inequality [24]: $\left|a_{i}+b_{i}\right|^{q_{i}} \leq 2^{H-1}\left(\left|a_{i}\right|^{q_{i}}+\left|b_{i}\right|^{q_{i}}\right)$, where $q_{i} \geq 0$, for all $i \in \mathbb{N}$ and $H=\max \left\{1, \sup _{i} q_{i}\right\}$, will be used in the sequel. 


\section{Main Results}

3.1. Prequasi-Norm on $\ell_{\beta}^{\left(\mathbf{p}_{\mathbf{n}}\right)}$. We investigate the sufficient conditions on the sequence space $\ell_{\beta}^{\left(p_{n}\right)}$ equipped with prequasi-norm $\tau$ to form prequasi-Banach and closed (sss).

Theorem 3. $\left(\ell_{\beta}^{\left(p_{n}\right)}\right)_{\tau}$, where $\tau(x)=\left[\sum_{k=0}^{\infty} \beta_{k}\left|x_{k}\right|^{p_{k}}\right]^{(1 / H)}$, for all $x \in \ell_{\beta}^{\left(p_{n}\right)}$, is a premodular (sss), if the following conditions are satisfied:

(a1) The sequence $\left(P_{n}\right) \in \mathbb{R}^{+\mathbb{N} \cap} \ell_{\infty}$ is increasing

(a2) Either $\left(\beta_{n}\right)$ is a monotonic decreasing or monotonic increasing such that there is $C \geq 1$ for which $\beta 2_{n}+1 \leq C \beta_{n}$

Proof. Firstly, we have to prove $\ell_{\beta}^{\left(p_{n}\right)}$ is a (sss):

(1-i) Let $x, \mathrm{y} \in \ell_{\beta}^{\left(p_{n}\right)}$. Since $\left(p_{n}\right)$ is bounded, we obtain

$$
\begin{aligned}
\tau(X+Y)= & {\left[\sum_{n=0}^{\infty} \beta_{n}\left|x_{n}+y_{n}\right|^{p_{n}}\right]^{(1 / H)} \leq\left[\sum_{n=0}^{\infty} \beta_{n}\left|x_{n}\right|^{p_{n}}\right]^{(1 / H)} } \\
& +\left[\sum_{n=0}^{\infty} \beta_{n}\left|y_{n}\right|^{p_{n}}\right]^{(1 / H)}=\tau(X)+\tau(Y)<\infty
\end{aligned}
$$

then $x+y \in \ell_{\beta}^{\left(p_{n}\right)}$.

(1-ii) Let $\lambda \in \mathbb{C}$ and $x \in \ell_{\beta}^{\left(p_{n}\right)}$. Since $\left(p_{n}\right)$ is bounded, we have

$$
\begin{aligned}
\tau(\lambda X) & =\left[\sum_{n=0}^{\infty} \beta_{n}\left|\lambda x_{n}\right|^{p_{n}}\right]^{(1 / H)} \leq_{n}^{\sup }|\lambda|\left(p_{n} / H\right)\left[\sum_{n=0}^{\infty} \beta_{n}\left|x_{n}\right|^{p_{n}}\right]^{(1 / H)} \\
& \leq a|\lambda| \tau(X)<\infty .
\end{aligned}
$$

(i) Then, $\lambda \mathrm{x} \in \ell_{\beta}^{\left(p_{n}\right)}$ Therefore, by using Parts (1-i) and (1ii) we have that the space $\ell_{\beta}^{\left(p_{n}\right)}$ is linear. Also, en $\epsilon$ $\ell_{\beta}^{\left(p_{n}\right)}$, for all $n \in \mathbb{N}$, since

$$
\tau\left(e_{n}\right)=\left[\sum_{i=0}^{\infty} \beta_{i}\left|e_{n}(i)\right|^{\mathbf{p}_{i}}\right]^{(1 / H)}=\left(\beta_{n}\right)^{(1 / H)} .
$$

(2) Let $\left|x_{n}\right| \leq\left|y_{n}\right|$, for all $n \in \mathbb{N}$ and $y \in \ell_{\beta}^{\left(p_{n}\right)}$. Since $\beta_{n}>0$, for all $n \in \mathbb{N}$, then $\tau(x)=$ $\left[\sum_{n=0}^{\infty} \beta_{n}\left|x_{n}\right|^{p_{n}}\right]^{(1 / H)} \leq\left[\sum_{n=0}^{\infty} \beta_{n}\left|y_{n}\right|^{p_{n}}\right]^{(1 / H)}=\tau(\mathrm{y})$ $<\infty$, we get $x \in \ell_{\beta}^{\left(p_{n}\right)}$.

(3) Let $\left(x_{n}\right) \in \ell_{\beta}^{\left(p_{n}\right)}$ and $\left(\beta_{n}\right)$ be an increasing sequence. There exists $C \geq 1$ such that $\beta 2_{n}+1 \leq \mathrm{C} \beta_{n}$ and $\left(p_{n}\right)$ be an increasing; then, we have

$$
\begin{aligned}
\tau\left(x_{[n / 2]}\right) & =\left[\sum_{n=0}^{\infty} \beta_{n}\left|x_{[n / 2]}\right|^{p_{n}}\right]^{(1 / H)}=\left[\sum_{n=0}^{\infty} \beta_{2 n}\left|x_{n}\right|^{p_{2 n}}+\sum_{n=0}^{\infty} \beta_{2 n+1}\left|x_{n}\right|^{p_{2 n+1}}\right]^{(1 / H)} \\
& \leq\left[\sum_{n=0}^{\infty} \beta_{2 n}\left|x_{n}\right|^{p_{n}}+\sum_{n=0}^{\infty} \beta_{2 n+1}\left|x_{n}\right|^{p_{n}}\right]^{(1 / H)} \leq(2 C)^{(1 / H)}\left[\sum_{n=0}^{\infty} \beta_{n}\left|x_{n}\right|^{p_{n}}\right]^{(1 / H)}=(2 C)^{(1 / H)} \tau\left(x_{n}\right),
\end{aligned}
$$

then $\left(x_{[n / 2]}\right) \in \ell_{\beta}^{\left(p_{n}\right)}$. Secondly, we show that the functional $\tau$ on $\ell_{\beta}^{\left(p_{n}\right)}$ is a premodular:

(i) Evidently, $\tau(x) \geq 0$ and $\tau(x)=0 \Leftrightarrow x=\theta$

(ii) There is a steady $a=\max \left\{1\right.$, supn $\left.|\lambda|\left(p_{n} / H\right)-1\right\} \geq 1$ such that $\tau(\lambda \mathrm{x}) \leq a|\lambda| \tau(x)$, for all $x \in \ell_{\beta}^{\left(p_{n}\right)}$ and $\lambda \in \mathbb{C}$

(iii) There exists $K \geq 1$ such that $\tau \quad(x+y) \leq \mathrm{K}$ $(\tau(x)+\tau(\mathrm{y}))$, for all $x, \mathrm{y} \in \ell_{\beta}^{\left(p_{n}\right)}$

(iv) Clearly, from the proof part (2) of Theorem 3 , the condition is clear since the weighted Nakano sequence space is solid

(v) It is obtained from (3) that $b_{0}=(2 C)^{(1 / H)} \geq 1$

(vi) It is clear that $\bar{F}=\ell_{\beta}^{\left(p_{n}\right)}$

(vii) There exists a steady $0<\xi \leq|\lambda|^{\left(p_{0} / H\right)-1}$, for $\lambda \neq 0$ or $\xi>0$, for $\lambda=0$ such that $\tau(\lambda, 0,0,0, \ldots) \geq \xi|\lambda| \tau(1$, $0,0,0, \ldots)$

Theorem 4. If conditions (a1) and (a2) are satisfied, then $\left(\ell_{\beta}^{\left(p_{n}\right)}\right)_{\tau}$, where $\tau(x)=\left[\sum_{k=0}^{\infty} \beta_{k}\left|x_{k}\right|^{p_{k}}\right]^{(1 / H)}$ for all $x \in \ell_{\beta}^{\left(p_{n}\right)}$, is a prequasi-Banach (sss).
Proof. Let the conditions be verified. By Theorem 3, the space $\left(\ell_{\beta}^{\left(p_{n}\right)}\right)_{\tau}$ is a premodular (sss). Therefore, from Theorem 2 , the space $\left(\ell_{\beta}^{\left(p_{n}\right)}\right)_{\tau}$ is a prequasi-normed (sss). To prove that $\left(\ell_{\beta}^{\left(p_{n}\right)}\right)_{\tau}$ is a prequasi-Banach (sss), suppose $\mathrm{x}_{\mathrm{n}}=\left(x_{k}^{n}\right)_{k=0}^{\infty}$ be a Cauchy sequence in $\left(\ell_{\beta}^{\left(p_{n}\right)}\right)_{\tau}$, then for every $\varepsilon \in(0,1)$, there exists a number $n_{0} \in \mathbb{N}$ such that, for all $n$, $m \geq n_{0}$, one has

$$
\tau\left(X^{n}-X^{m}\right)=\left[\sum_{j=0}^{\infty} \beta_{j}\left|x_{j}^{n}-x_{j}^{m}\right|^{\mathbf{p}_{j}}\right]^{(1 / H)}<\varepsilon .
$$

Hence, for $n, m \geq n_{0}$ and $j \in \mathbb{N}$, we get $\left|x_{j}^{n}-x_{j}^{m}\right|<\varepsilon$.

So, $\left(x_{j}^{m}\right)$ is a Cauchy sequence in $\mathbb{C}$ for fixed $j \in \mathbb{N}$, and this gives $\lim x_{j}^{m}=x_{j}^{0}$ for $j \in \mathbb{N}$. Hence, $\tau\left(\mathrm{x}_{\mathrm{n}}-\mathrm{x}_{0}\right)<\varepsilon$, for all $n \geq n_{0}$. Finally, to prove that $\mathrm{x}_{0} \in \ell_{\beta}^{\left(p_{n}\right)}$, we have $\tau\left(\mathrm{x}_{0}\right)=\tau$ $\left(\mathrm{x}_{0}-\mathrm{x}_{\mathrm{n}}+\mathrm{x}_{\mathrm{n}}\right) \leq \tau\left(\mathrm{x}_{\mathrm{n}}-\mathrm{x}_{0}\right)+\tau\left(\mathrm{x}_{\mathrm{n}}\right)<\infty$.

So, $\mathrm{x}_{0} \in \ell_{\beta}^{\left(p_{n}\right)}$. This means that $\left(\ell_{\beta}^{\left(p_{n}\right)}\right)_{\tau}$ is a prequasiBanach (sss).

Theorem 5. If conditions (a1) and (a2) are satisfied, then $\left(\ell_{\beta}^{\left(p_{n}\right)}\right)_{\tau}$, where $\tau(x)=\left[\sum_{k=0}^{\infty} \beta_{k}\left|x_{k}\right|^{p_{k}}\right]^{(1 / H)}$, for all $x \in \ell_{\beta}^{\left(p_{n}\right)}$, is a prequasi-closed (sss). 
Proof. Let the conditions be verified. By Theorem 3, the space $\left(\ell_{\beta}^{\left(p_{n}\right)}\right)_{\tau}$ is a premodular (sss). Therefore, from Theorem 2 , the space $\left(\ell_{\beta}^{\left(p_{n}\right)}\right)_{\tau}$ is a prequasi-normed (sss). To prove that $\left(\ell_{\beta}^{\left(p_{n}\right)}\right)_{\tau}$ is a prequasi-closed (sss), suppose $X^{n}=\left(x_{k}^{n}\right)_{k=0}^{\infty} \in\left(\ell_{\beta}^{\left(p_{n}\right)}\right)_{\tau}$ and $\operatorname{limn} \longrightarrow \infty \tau\left(X^{n}-X^{0}\right)=0$, then for every $\varepsilon \in(0,1)$, there exists a number $n_{0} \in \mathbb{N}$ such that for all $n \geq n_{0}$, one has

$$
\varepsilon>\tau\left(X^{n}-X^{0}\right)=\left[\sum_{j=0}^{\infty} \beta_{j}\left|x_{j}^{n}-x_{j}^{0}\right|^{p_{j}}\right]^{(1 / H)} \geq\left[\beta_{j}\left|x_{j}^{n}-x_{j}^{0}\right|^{p_{j}}\right]^{(1 / H)} .
$$

Hence, for $n \geq n_{0}$ and $j \in \mathbb{N}$, we get $\left|x_{j}^{n}-x_{j}^{0}\right|<\varepsilon$.

So, $\left(x_{j}^{n}\right)$ is a convergent sequence in $\mathbb{C}$ for fixed $j \in \mathbb{N}$, and this gives limm $\longrightarrow \infty x_{j}^{m}=x_{j}^{0}$ for fixed $j \in \mathbb{N}$. Finally, to prove that $\mathrm{x}_{0} \in \ell_{\beta}^{\left(p_{n}\right)}$, we have $\tau$ $\left(\mathrm{x}_{0}\right)=\tau\left(\mathrm{x}_{0}-\mathrm{x}_{\mathrm{n}}+\mathrm{x}_{\mathrm{n}}\right) \leq \tau\left(\mathrm{x}_{\mathrm{n}}-\mathrm{x}_{0}\right)+\tau\left(\mathrm{x}_{\mathrm{n}}\right)<\infty$.

So, $\mathrm{x}_{0} \in \ell_{\beta}^{\left(p_{n}\right)}$. This means that $\left(\ell_{\beta}^{\left(p_{n}\right)}\right)_{\tau}$ is a prequasiclosed (sss).

3.2. Bounded Multiplication Operator on $\ell_{\beta}^{\left(p_{n}\right)}$. Here after, we investigate some topological and geometric structure of the multiplication operator acting on $\ell_{\beta}^{\left(p_{n}\right)}$. In this section, we examine the sufficient conditions on the sequence space $\ell_{\beta}^{\left(p_{n}\right)}$ equipped with prequasi-norm $\tau$ such that the multiplication operator defined on $\left(\ell_{\beta}^{\left(p_{n}\right)}\right)_{\tau}\left(\ell_{\beta}^{\left(\mathrm{p}_{\mathrm{n}}\right)}\right)_{\tau}$ is bounded and isometry.

Definition 6. Let $\kappa \in \mathbb{C}^{\mathbb{N}} \cap \ell_{\infty}$ and $\mathrm{W}_{\tau}$ be a prequasi-normed (sss). An operator $\mathrm{V}_{\kappa}: \mathrm{W}_{\tau} \longrightarrow \mathrm{W}_{\tau}$ is called multiplication operator if $\mathrm{V}_{\kappa} \omega=\kappa \omega=\left(K_{r} \omega_{r}\right)_{r=0}^{\infty} \in \mathrm{W}$, for all $\omega \in \mathrm{W}$. If $\mathrm{V} \kappa \in$ $B(W)$, we name it a multiplication operator generated by $\kappa$.

Theorem 6. Let $\kappa \in \mathbb{C}^{\mathbb{N}}$ and conditions (a1) and (a2) be satisfied; then, $\kappa \in \ell \infty$, if and only if $V_{K} \in B\left(\ell_{\beta}^{\left(p_{n}\right)}\right)_{\tau}$, where $\tau(x)=\left[\sum_{k=0}^{\infty} \beta_{k}\left|x_{k}\right|^{p_{k}}\right]^{(1 / H)}$, for all $x \in \ell_{\beta}^{\left(p_{n}\right)}$.

Proof. Let the conditions be satisfied. Assume $\kappa \in \ell \infty$. Therefore, there is $\varepsilon>0$ with $\left|\kappa_{r}\right| \leq \varepsilon$, for every $r \in \mathbb{N}$. For $x \in$ $\left(\ell_{\beta}^{\left(p_{n}\right)}\right)_{\tau}$, since $\left(p_{r}\right)$ is bounded from above with $p_{r}>0$, for all $r \in \mathbb{N}$, then

$$
\begin{aligned}
\tau\left(V_{K} X\right) & =\tau(K X)=\tau\left(\left(K_{r} X_{r}\right)_{r=0}^{\infty}\right)=\left[\sum_{r=0}^{\infty} \beta_{r}\left|\kappa_{r} x_{r}\right|^{p_{r}}\right]^{(1 / H)} \\
& \leq\left[\sum_{r=0}^{\infty} \beta_{r}\left|\varepsilon x_{r}\right|^{p_{r}}\right]^{(1 / H)} \leq \sup _{r}^{\varepsilon\left(p_{r} / H\right)}\left[\sum_{r=0}^{\infty} \beta_{r}\left|x_{r}\right|^{p_{r}}\right]^{(1 / H)} \\
& =D \tau(x) .
\end{aligned}
$$

This gives $\mathrm{V}_{\kappa} \in B\left(\ell_{\beta}^{\left(p_{n}\right)}\right)_{\tau}$. Conversely, assume that $\mathrm{V}_{\kappa} \in$ $B\left(\ell_{\beta}^{\left(p_{n}\right)}\right)_{\tau}$. Let us assume $\kappa \notin \ell \infty$; hence, for each $j \in \mathbb{N}$, there is $i_{j} \in \mathbb{N}$ such that $\kappa_{i_{j}}>j$. Since $\left(p_{n}\right) \in \mathbb{R}^{+\mathbb{N}} \cap \ell \infty$ is increasing, we have

$$
\begin{aligned}
\tau\left(V_{K} e_{i_{j}}\right) & =\tau\left(K e_{i_{j}}\right)=\tau\left(K_{r}\left(e_{i_{j}}\right)_{\tau}\right)_{r=0}^{\infty} \\
& =\left[\sum_{r=0}^{\infty} \beta_{r}\left|\kappa_{r}\left(e_{i_{j}}\right)_{r}\right|^{p_{r}}\right]^{(1 / H)}=\left[\beta_{i_{j}} \mid \kappa_{i_{j}}\right]^{\left(p_{i j} / H\right)} \\
& >\left[\beta_{i_{j}}|j|\right]^{\left(p_{i j} / H\right)}=\left[\beta_{i_{j}}|j|\right]^{\left(p_{i j} / H\right)} \tau\left(e_{i_{j}}\right) .
\end{aligned}
$$

This shows that $\mathrm{V}_{\kappa} \in B\left(\ell_{\beta}^{\left(p_{n}\right)}\right)_{\tau}$. Therefore, $\kappa \in \ell \infty$.

Theorem 7. Pick up $\kappa \in \mathbb{C}^{\mathbb{N}}$ and $\left(\ell_{\beta_{n}}^{\left(p_{n}\right)}\right)_{\tau}$ be a prequasinormed (sss), with $\tau(x)=\left[\sum_{k=0}^{\infty} \beta_{k}\left|x_{k}\right|^{p_{k}}\right]^{(1 / H)}$, for all $x \in \ell_{\beta}^{\left(p_{n}\right)}$. Then, $\left|\kappa_{r}\right|=1$, for all $r \in \mathbb{N}$, if and only if, $V \kappa$ is an isometry.

Proof. Suppose $\left|\kappa_{\mathrm{r}}\right|=1$, for all $r \in \mathbb{N}$. Hence,

$$
\begin{aligned}
\tau\left(V_{K} X\right) & =\tau(K X)=\tau\left(K_{r} X_{r}\right)_{r=0}^{\infty}=\left[\sum_{r=0}^{\infty} \beta_{r}\left|\kappa_{r} x_{r}\right|^{p_{r}}\right]^{(1 / H)} \\
& =\left[\sum_{r=0}^{\infty} \beta_{r}\left|x_{r}\right|^{p_{r}}\right]^{(1 / H)}=\tau(x),
\end{aligned}
$$

for all $x \in\left(\ell_{\beta}^{\left(p_{n}\right)}\right)_{\tau}$. Therefore, $\mathrm{V}_{\kappa}$ is an isometry. Conversely, Assume that $|\kappa \mathrm{i}|<1$ for some $i=i 0$. Since $\left(p_{r}\right) \in \mathbb{R}^{+\mathbb{N}} \cap \ell \infty$ is increasing, we obtain

$$
\begin{aligned}
\tau\left(V e_{i_{0}}\right) & =\tau\left(K e_{i_{0}}\right)=\tau\left(K_{r}\left(e_{i_{0}}\right)_{r}\right)_{r=0}^{\infty}=\left[\sum_{r=0}^{\infty} \beta_{r}\left|\kappa_{r}\left(e_{i_{0}}\right)_{r}\right|^{p_{r}}\right]^{(1 / H)} \\
& <\left[\sum_{r=0}^{\infty} \beta_{r}\left|\left(e_{i_{0}}\right)_{r}\right|^{p_{r}}\right]^{(1 / H)}=\tau\left(e_{i_{0}}\right) .
\end{aligned}
$$

When $\left|\kappa_{i_{0}}\right|>1$, we can prove that $\tau\left(V_{K} e_{i_{0}}\right)>\tau\left(e_{i_{0}}\right)$. Therefore, in both cases, we have a contradiction. So, $\left|\kappa_{\mathrm{r}}\right|=1$, for every $r \in \mathbb{N}$.

3.3. Approximable Multiplication Operator on $\ell_{\beta}^{\left(p_{n}\right)}$. In this section, we introduce the sufficient conditions on the sequence space $\ell_{\beta}^{\left(p_{n}\right)}$ equipped with prequasi-norm $\tau$ such that the multiplication operator defined on $\left(\ell_{\beta}^{\left(p_{n}\right)}\right)_{\tau}$ is an approximable and compact. By card (A), we indicate the cardinality of the set A.

Theorem 8. If $\kappa \in \mathbb{C}^{\mathbb{N}}$ and $\left(\left(\ell_{\beta}^{\left(p_{n}\right)}\right)_{\tau}\right)$ is a prequasi-normed (sss), where $\tau(x)=\left[\sum_{k=0}^{\infty} \beta_{k}\left|x_{k}\right|^{p_{k}}\right]^{(1 / H)}$, for all $x \in \ell_{\beta}^{\left(p_{n}\right)}$, then, $V_{\kappa} \in \Upsilon\left(\left(\ell_{\beta}^{\left(p_{n}\right)}\right)_{\tau}\right)$ if and only if $\left(K_{r}\right)_{r=0}^{\infty} \in c_{0}$.

Proof. Assume that $\mathrm{V}_{\kappa} \in \Upsilon\left(\left(\ell_{\beta}^{\left(p_{n}\right)}\right)_{\tau}\right)$. Therefore, $\mathrm{V}_{\kappa} \in$ $B B_{c}\left(\ell_{\beta}^{\left(p_{n}\right)}\right)_{\tau}$, to prove that the sequence $\left(K_{r}\right)_{r=0}^{\infty}$ belongs to $c_{0}$. Suppose $\left(K_{r}\right)_{r=0}^{\infty} \notin \mathrm{c}_{0}$. Hence, there is $\delta>0$ such that the set $A_{\delta}=\left\{\mathrm{r} \in \mathbb{N}:\left|\kappa_{\mathrm{r}}\right| \geq \delta\right\}$ has card $\left(A_{\delta}\right)=\infty$. Assume ai $\in A_{\delta}$, for all $i \in \mathbb{N}$. Hence, $\left\{e_{a_{i}}:\right.$ ai $\left.\in \mathrm{A} \delta\right\}$ is an infinite bounded set in $\left(\ell_{\beta}^{\left(p_{n}\right)}\right)_{\tau}$. Let 


$$
\begin{aligned}
\tau\left(V_{K} e_{a_{i}}-V_{K} e_{a_{j}}\right) & =\tau\left(K e_{a_{i}}-K e_{a_{i}}\right)=\tau\left(\kappa_{r}\left(\left(e_{a_{i}}\right)_{r}-\left(e_{a_{i}}\right)_{r}\right)\right)_{r=0}^{\infty} \\
& =\left[\sum_{r=0}^{\infty} \beta_{r}\left|\kappa_{r}\left(\left(e_{a_{i}}\right)_{r}-\left(e_{a_{j}}\right)_{r}\right)\right|^{p_{r}}\right]^{(1 / H)} \\
& \geq\left[\sum_{r=0}^{\infty} \beta_{r}\left|\delta\left(\left(e_{a_{i}}\right)_{r}-\left(e_{a_{j}}\right)_{r}\right)\right|^{p_{r}}\right]^{(1 / H)} \\
& \geq{ }_{r} \delta^{\left(p_{r} / H\right)} \tau\left(e_{a_{i}}-e_{a_{j}}\right),
\end{aligned}
$$

for all $a_{i}, a_{j} \in \mathrm{A} \delta$. This shows $\left\{e_{a_{i}}: a_{i} \in \mathrm{B} \delta\right\} \in \ell \infty$ which cannot have a convergent subsequence under $\mathrm{V}_{\kappa}$. This proves that $\mathrm{V}_{\mathrm{k}} \notin B_{c}\left(\ell_{\beta}^{\left(p_{n}\right)}\right)_{\tau}$. Then, $\mathrm{V}_{\mathrm{k}} \notin \Upsilon\left(\left(\ell_{\beta}^{\left(p_{n}\right)}\right)_{\tau}\right)$, this gives a contradiction. So, limi $\longrightarrow \infty \kappa_{\mathrm{i}}=0$. Conversely, let limi $\longrightarrow \infty \kappa_{\mathrm{i}}=0$. Then, for each $\delta>0$, the set $A_{\delta}=\left\{\mathrm{i} \in \mathbb{N}:\left|\kappa_{\mathrm{i}}\right| \geq \delta\right\}$ has card $(\mathrm{A} \delta)<\infty$. Hence, for every $\delta>0$, the space $\left(\left(\ell_{\beta}^{\left(p_{n}\right)}\right)_{\tau}\right)_{A_{\delta}}=\left\{x=(\mathrm{xi}) \in \mathbb{C}^{A_{\delta}}\right\}$ is finite dimensional. Then, $\mathrm{V} \kappa \mid\left(\left(\ell_{\beta}^{\left(p_{n}\right)}\right)_{\tau}\right)_{A_{\delta}}$ is a finite rank operator. For every $i \in \mathbb{N}$, dene $\kappa \mathrm{i} \in \mathbb{C}^{\mathbb{N}}$ by $(\kappa i) j=\left[\begin{array}{cc}\kappa_{j}, & j \in A_{(1 / i)} \\ 0, & \text { otherwise. }\end{array}\right]$

It is clear that $V_{\kappa_{i}}$ has rank $\left(V_{\kappa_{i}}\right)<\infty$ as $\operatorname{dim}$ $\left(\left(\ell_{\beta}^{\left(p_{n}\right)}\right)_{\tau}\right)_{A_{(1 / i)}}<\infty$, for each $i \in \mathbb{N}$. Therefore, since $\left(p_{n}\right) \in$ $\mathbb{R}^{+\infty} \cap$ lo is increasing, we obtain

$$
\begin{aligned}
\tau\left(\left(V_{k}-V_{k_{i}}\right) X\right) \tau\left(\left(k_{j}-\left(k_{i}\right) j\right) X_{j}\right) j==_{0}^{\infty} & \left.=\left[\sum_{j=0}^{\infty} \beta_{j}\left|\left(\kappa_{j}-\left(\kappa_{i}\right)_{j}\right) x_{j}\right|^{p_{j}}\right]^{(1 / H)}\left[\sum_{j=0, j \in A_{(1 / i)} \beta_{j}\left|\left(\kappa_{j}-\left(\kappa_{i}\right)_{j}\right) x_{j}\right|^{p_{j}}}^{\infty}+\sum_{\left.j=0, j \in A_{(1 / i)} \beta_{j}\left|\left(\kappa_{i}-\left(\kappa_{i}\right)_{j}\right) x_{j}\right|^{p_{j}}\right]^{(1 / H)}}^{\infty}\right]\left[\sum_{j=0, j \notin A_{(1 / i)}}^{\infty} \beta_{j}\left|\kappa_{j} x_{j}\right|^{p_{j}}\right]_{j=0, j \notin A_{(1 / i)}}^{(1 / H)} \beta_{j}\left|x_{i}\right|^{p_{j}}\right]^{\left(p_{j} / H\right)} \\
> & \sup _{j}^{\infty}\left(\frac{1}{j}\right)^{\left(p_{j} / H\right)} \tau(x)=\left(\frac{1}{j}\right)^{\left(p_{0} / H\right)} \tau(x) .
\end{aligned}
$$

This implies that $\left\|B_{c}-V_{K_{i}}\right\| \leq(1 / j)^{\left(p_{0} / H\right)}$ and that $\mathrm{V}_{\kappa}$ is a limit of finite rank operators. Therefore, $V_{\kappa}$ is an approximable operator.

Theorem 9. Let $\kappa \in \mathbb{C}^{\mathbb{N}}$ and $\left(\ell_{\beta}^{\left(p_{n}\right)}\right)_{\tau}$ be a prequasi-normed (sss), where $\tau(x)=\left[\sum_{r=0}^{\infty} \beta_{r}\left|x_{r}\right|^{p_{r}}\right]^{(1 / H)}$, for all $x \in\left(\ell_{\beta}^{\left(p_{n}\right)}\right)_{\tau}$. Then, $V_{\kappa} \in B_{c}\left(\ell_{\beta}^{\left(p_{n}\right)}\right)_{\tau}$, if and only if, $\left(K_{i}\right)_{i=0}^{\infty} \in c_{0}$.

Proof. It is simple so overlooked.

Corollary 1. If $\kappa \in \mathbb{C}^{\mathbb{N}}$, conditions (a1) and (a2) are satisfied, then $B_{c}\left(\left(\ell_{\beta}^{\left(p_{n}\right)}\right)_{\tau}\right)$ B $B\left(\left(\ell_{\beta_{n}}^{\left(p_{n}\right)}\right)_{\tau}\right)$, where $\tau(x)=\left[\sum_{r=0}^{\infty} \beta_{r}\left|x_{r}\right|^{p_{r}}\right]^{\left(1 / H^{\dagger}\right)}$, for all $x \in \ell_{\beta}^{\left(\beta_{n}\right)}$.

Proof. Since I is a multiplication operator on $\left(l_{\beta}^{\left(P_{n}\right)}\right)_{\tau}$ generated by $\kappa=(1,1, \ldots)$, therefore, $I \notin B_{c}\left(\ell_{\beta}^{\left(p_{n}\right)}\right)_{\tau}^{\beta}$ and $\mathrm{I} \in$ $B\left(\left(\ell_{\beta}^{\left(p_{n}\right)}\right)_{\tau}\right)$.
3.4. Fredholm Multiplication Operator on $\ell_{\beta}^{\left(\mathbf{p}_{\mathbf{n}}\right)}$. In this section, we give the sufficient conditions on the sequence space $\ell_{\beta}^{\left(p_{n}\right)}$ equipped with prequasi-norm $\tau$ such that the multiplication operator defined on $\left(l_{\beta}^{\left(P_{n}\right)}\right)_{\tau}$ has closed range, invertible, and Fredholm.

Theorem 10. If $\kappa \in \mathbb{C}^{\mathbb{N}},\left(\ell_{\beta_{n}}^{\left(p_{n}\right)}\right)_{\tau}$ is prequasi-Banach (sss), where $\tau(x)=\left[\sum_{r=0}^{\infty} \beta_{r}\left|x_{r}\right|^{p_{r}}\right]^{\left({ }^{(1 / H)}\right.}$, for all $x \in\left(\ell_{\beta}^{\left(p_{n}\right)}\right)_{\tau}$, then $\kappa$ is bounded away from zero on $(\operatorname{ker}(\kappa))$, if and only if, $R(V \kappa)$ is closed.

Proof. Let the sufficient condition be satisfied. Therefore, there is $\varepsilon>0$ with $|\kappa i| \geq \varepsilon$, for all $i \in(\operatorname{ker}(\kappa)) c$, to show $\mathrm{R}(\mathrm{V} \kappa)$ is closed. Assume $d$ be a limit point of $\mathrm{R}(\mathrm{V} \kappa)$. Therefore, there is $\mathrm{V}_{\kappa} \mathrm{xi}$ in $\left(\ell_{\beta}^{\left(p_{n}\right)}\right)_{\tau}$, for all $i \in \mathbb{N}$ such that $\lim i \longrightarrow \infty$ $\mathrm{V} \kappa \mathrm{xi}=d$. Obviously, $\left(\mathrm{V}_{\kappa} \mathrm{xi}\right)$ is a Cauchy sequence. Since $\left(p_{n}\right)$ $\in \mathbb{R}^{+\mathbb{N}} \cap$ lo is increasing, one has 


$$
\begin{aligned}
\tau\left(V_{k} x_{i}-V_{k} x_{j}\right) & =\left[\sum_{r=0}^{\infty} \beta_{r}\left|\kappa_{r}\left(x_{i}\right)_{r}-\kappa_{r}\left(x_{j}\right)_{r}\right|^{p_{r}}\right]^{(1 / H)}=\left[\sum_{r=0, r \in(\operatorname{ker}(\kappa))^{c}}^{\infty} \beta_{r}\left|\kappa_{r}\left(x_{i}\right)_{r}-\kappa_{r}\left(x_{j}\right)_{r}\right|^{p_{r}}+\sum_{r=0, r \notin(\operatorname{ker}(\kappa))^{c}}^{\infty} \beta_{r}\left|\kappa_{r}\left(x_{i}\right)_{r}-\kappa_{r}\left(x_{j}\right)_{r}\right|^{p_{r}}\right]^{(1 / H)} \\
& \geq\left[\sum_{r=0, r \in(\operatorname{ker}(\kappa))^{c}}^{\infty} \beta_{r}\left|\kappa_{r}\left(x_{i}\right)_{r}-\kappa_{r}\left(x_{j}\right)_{r}\right|^{p_{r}}\right]^{(1 / H)}=\left[\sum_{r=0}^{\infty} \beta_{r}\left|\kappa_{r}\left(\left(y_{i}\right)_{r}-\left(y_{j}\right)_{r}\right)\right|^{p_{r}}\right]^{\frac{1}{H}}>_{r}^{\inf _{\varepsilon}\left(p_{r} / H\right)}\left[\sum_{r=0}^{\infty} \beta_{r}\left|\left(y_{i}\right)_{r}-\left(y_{j}\right)_{r}\right|^{p_{r}}\right]^{(1 / H)},
\end{aligned}
$$

where

$$
\left(y_{i}\right)_{r}=\left[\begin{array}{ll}
\left(x_{i}\right)_{r}, & r \in(\operatorname{ker}(\kappa))^{c}, \\
0, & r \notin(\operatorname{ker}(\kappa))^{c} .
\end{array}\right.
$$

This shows that (yi) is a Cauchy sequence in $\left(\ell_{\beta}^{\left(p_{n}\right)}\right)_{\tau}$. Since $\left(\ell_{\beta}^{\left(p_{n}\right)}\right)_{\tau}$ is complete, there is $x \in \in\left(\ell_{\beta}^{\left(p_{n}\right)}\right)_{\tau}$ such that limi $\longrightarrow \infty y i=x$. Since $V_{\kappa}$ is continuous, then limi $\longrightarrow \infty$ $\mathrm{V}_{\kappa} \mathrm{yi}=\mathrm{V}_{\kappa} \mathrm{x}$. However, $\operatorname{limi} \longrightarrow \infty \quad \mathrm{V}_{\kappa} \mathrm{xi}=\lim i \longrightarrow \infty$ $\mathrm{V}_{\kappa} \mathrm{yi}=d$. Hence, $\mathrm{V}_{\kappa} \mathrm{x}=d$. Therefore, $d \in \mathrm{R}(\mathrm{V} \kappa)$. This shows that $\mathrm{R}(\mathrm{V} \kappa)$ is closed. Conversely, let $\mathrm{R}(\mathrm{V} \kappa)$ be closed. Therefore, $V_{\kappa}$ be bounded away from zero on

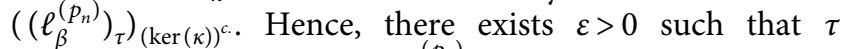
$\left(V_{\kappa} x\right) \geq \varepsilon \tau(x)$, for all $x \in\left(\left(\ell_{\beta}^{\left(p_{n}\right)}\right)_{\tau}\right)_{(\operatorname{ker}(\kappa))^{c}}$.

Assume $B=\left\{\mathrm{r} \in(\operatorname{ker}(\kappa))^{c}:\left|\kappa_{\mathrm{r}}\right|>\right\}$. If $\mathrm{B} \neq \phi$, then for $i_{0} \in \mathrm{B}$, we obtain

$$
\begin{aligned}
& \tau\left(V_{K} e_{n_{0}}\right)=\tau\left(K_{r}\left(e_{n_{0}}\right)_{r}\right)_{r=0}^{\infty}=\left[\sum_{r=0}^{\infty} \beta_{r}\left|\kappa_{r}\left(e_{n_{0}}\right)_{r}\right|^{p_{r}}\right]^{(1 / H)} \\
& <\left[\sum_{r=0}^{\infty} \beta_{r}\left|\varepsilon\left(e_{n_{0}}\right)_{r}\right| p_{r}\right]^{(1 / H)} \\
& \leq \sup \varepsilon^{\left(p_{r} / H\right)}\left[\sum_{r=0}^{\infty} \beta_{r}\left|\left(e_{n_{0}}\right)_{r}\right|^{p_{r}}\right]^{(1 / H)} \\
& =\sup \varepsilon^{\left(p_{r} / H\right)}{ }_{r} \tau\left(e_{n_{0}}\right) \text {, }
\end{aligned}
$$

and this gives contradiction. So, $B=\phi$ such that $\left|\kappa_{r}\right| \geq \varepsilon$, for all $r \in(\operatorname{ker}(\kappa))^{c}$. This completes the proof of the theorem.

Theorem 11. If $\kappa \in \mathbb{C}^{\mathbb{N}}$ and $\left(\ell_{\beta}^{\left(p_{n}\right)}\right)_{\tau}$ is a prequasi-Banach (sss), with $\tau(\omega)=\left.\sum_{r=0}^{\infty} \beta_{r}\left|\omega_{r}\right|^{p_{r}}\right|^{(1 / H)}$, for all $(\omega) \in \ell_{\beta}^{\left(p_{n}\right)}$, there are $b>0$ and $B>0$ such that $b<\left|\kappa_{r}\right|<B$, for all $r \in \mathbb{N}$, if and only if, $V_{\kappa} \in B\left(\ell_{\beta}^{\left(p_{n}\right)}\right)_{\tau}$ is invertible.

Proof. Let the conditions be verified. Define $\gamma \in \mathrm{CN}$ by $\gamma \mathrm{r}=\left(1 / \kappa_{r}\right)$. From Theorem 6 , we have $\mathrm{V}_{\kappa}, \mathrm{V}_{\gamma} \in B\left(\ell_{\beta}^{\left(p_{n}\right)}\right)_{\tau}$ and $\mathrm{V}_{\kappa} \cdot \mathrm{V}_{\gamma}=\mathrm{V}_{\gamma} \cdot \mathrm{V}_{\kappa}=\mathrm{I}$. Then, $\mathrm{V}_{\gamma}$ is the inverse of $\mathrm{V}_{\kappa}$. Conversely, let $\mathrm{V}_{\kappa}$ be invertible. Hence, $R\left(\mathrm{~V}_{\kappa}\right)=\left(\left(\left(\ell_{\beta}^{\left({ }_{p}\right)}\right)_{\tau}\right)\right)_{\mathbb{N}}$. This implies $R\left(\mathrm{~V}_{\kappa}\right)$ is closed. From Theorem 10, there is $b>0$ such that $\left|\kappa_{r}\right| \geq b$, for all $r \in(\operatorname{ker}(\kappa))$ c. Now, $\operatorname{ker}(\kappa)=\phi$, else $\kappa_{\mathrm{r}_{\mathrm{o}}}=0$, for several $\mathrm{r}_{\mathrm{o}} \in \mathbb{N}$, we get $\mathrm{e}_{\mathrm{r}_{\mathrm{o}}} \in \operatorname{ker}\left(\mathrm{V}_{\kappa}\right)$. This gives a contradiction, since ker $\left(\mathrm{V}_{\kappa}\right)$ is trivial. So, $\left|\kappa_{r}\right| \geq b$, for all $r \in$ $\mathbb{N}$. Since $\mathrm{V} \kappa$ is bounded, hence from Theorem 6 , there is $B>0$ such that $\left|\kappa_{r}\right| \leq \mathrm{B}$, for all $r \in \mathbb{N}$. Therefore, we have shown that $b \leq\left|\kappa_{r}\right| \leq \mathrm{B}$, for all $r \in \mathbb{N}$.

Theorem 12. If $\kappa \in \mathbb{C}^{\mathbb{N}}$ and $\left(\ell_{\beta}^{\left(p_{n}\right)}\right)_{\tau}$ is a prequasi-Banach (sss), where $\tau(\omega)=\left.\sum_{r=0}^{\infty} \beta_{r}\left|\omega_{r}\right|^{p_{r}}\right|^{(1 / H)}$, for all $(\omega) \in \ell_{\beta}^{\left(p_{n}\right)}$, then $V \kappa \in B\left(\ell_{\beta}^{\left(p_{n}\right)}\right)_{\tau}$ is Fredholm operator, if and only if, (i) card $(\operatorname{ker}(\kappa))<\infty$ and $(\mathrm{ii})\left|\kappa_{r}\right| \geq \varepsilon$, for all $r \in(\operatorname{ker}(\kappa)) c$.

Proof. Let $\mathrm{V}_{\kappa}$ be Fredholm. If $\operatorname{card}(\operatorname{ker}(\kappa))=\infty$, hence en $\epsilon$ $\operatorname{ker}(\mathrm{V} \kappa)$, for all $n \in \operatorname{ker}(\kappa)$. Since en's are linearly independent, this gives card $(\operatorname{ker}(\mathrm{V} \kappa))=\infty$. This is a contradiction. Therefore, card $(\operatorname{ker}(\kappa))<\infty$. By Theorem 10, condition (ii) is satisfied. Next, if the necessary conditions are verified, to show that $V_{\kappa}$ is Fredholm. From Theorem 10, condition (ii) gives that $\mathrm{R}\left(\mathrm{V}_{\kappa}\right)$ is closed. Condition (i) indicates that $\operatorname{dim}\left(\operatorname{ker}\left(\mathrm{V}_{\kappa}\right)\right)<\infty$ and $\operatorname{dim}\left(\left(\mathrm{R}\left(\mathrm{V}_{\kappa}\right)\right) \mathrm{c}\right)<\infty$. Therefore, $V_{\kappa}$ is Fredholm.

\section{Data Availability}

No data were used to support this study.

\section{Conflicts of Interest}

The authors declare that they have no competing interests.

\section{Authors' Contributions}

All authors contributed equally to the writing of this paper. All authors read and approved the final manuscript.

\section{Acknowledgments}

This work was funded by the University of Jeddah, Saudi Arabia, under Grant no. UJ-02-054-DR. The authors, therefore, acknowledge with thanks the University technical and financial support.

\section{References}

[1] A. Pietsch, "Small ideals of operators," Studia Mathematica, vol. 51, no. 3, pp. 265-267, 1974.

[2] B. M. Makarov and N. Faried, "Some properties of operator ideals constructed by s numbers (In Russian)," in Theory of Operators in Functional Spaces, pp. 206-211, Academy of Science, Siberian section, Novosibirsk, Russia, 1977.

[3] N. Faried and A. A. Bakery, "Mappings of type orlicz and generalized cesáro sequence space," Journal of Inequalities and Applications, vol. 1, 2013.

[4] A. Pietsch, Operator Ideals, North-Holland Publishing Company, Amsterdam, NY, USA, 1980.

[5] A. A. Bakery and A. R. Abou Elmatty, "Pre-quasi simple Banach operator ideal generated by $s$ - numbers," Journal of Function Spaces, Article ID 9164781, 11 pages, 2020. 
[6] S. T. Chen, "Geometry of orlicz spaces," Dissertations Mathematics, vol. 356, pp. 1-204, 1996.

[7] Y. A. Cui and H. Hudzik, "On the Banach-saks and weak Banach-saks properties of some Banach sequence spaces," Acta Scientiarum Mathematicarum, vol. 65, pp. 179-187, 1999.

[8] W. Orlicz, "Über konjugierte Exponentenfolgen," Studia Mathematica, vol. 3, no. 1, pp. 200-211, 1931.

[9] V. Klee, "Summability in $\ell\left(p_{11}, p_{21}, \ldots\right)$ spaces," Studia Mathematica, vol. 25, pp. 277-280, 1965.

[10] K. Sundaresan, "Uniform convexity of Banach spaces 1 ( \{p_ \{i\}\})," Studia Mathematica, vol. 39, no. 3, pp. 227-231, 1971.

[11] M. B. Abrahmse, "Multiplication operators," in Lecture Notes in Mathematics, pp. 17-36, Springer-Verlag, Berlin, Germany, 1978.

[12] A. Sharma, K. Raj, and S. K. Sharma, "Products of multiplication composition and differentiation operators from $H^{\infty}$ weighted Bloch spaces," Indian Journal of Mathematics, vol. 54, pp. 159-179, 2012.

[13] R. K. Singh and J. S. Manhas, Composition Operators on Function Spaces, Elsevier, Amsterdam, Netherlands, 1993.

[14] H. Takagi and K. Yokouchi, "Multiplication and composition operators between two L $\{\mathrm{P}\}$-spaces," Function Spaces, vol. 232, pp. 321-338, 1999.

[15] M. İlkhan, S. Demiriz, and E. E. Kara, "Multiplication operators on Cesáro second order function spaces," Positivity, vol. 24, pp. 605-614, 2020.

[16] E. E. Kara and M. Basarir, "On compact operators and some Euler B(m)-difference sequence spaces," Journal of Mathematical Analysis and Applications, vol. 2, no. 379, pp. 499-511, 2011.

[17] A. Pietsch, Eigenvalues and S-Numbers, Cambridge University Press, New York. NY, USA, 1986.

[18] N. Faried and A. A. Bakery, "Small operator ideals formed by s numbers on generalized Cesáro and Orlicz sequence spaces," Journal of Inequalities and Applications, vol. 1, 2018.

[19] M. Mursaleen and A. K. Noman, "Compactness by the Hausdorff measure of noncompactness," Nonlinear Analysis: Theory, Methods \& Applications, vol. 73, no. 8, pp. 2541-2557, 2010.

[20] M. Mursaleen and A. K. Noman, "Compactness of matrix operators on some new difference sequence spaces," Linear Algebra and Its Applications, vol. 436, no. 1, pp. 41-52, 2012.

[21] B. S. Komal, S. Pandoh, and K. Raj, "Multiplication operators on Cesáro sequence spaces," Demonstratio Mathematica, vol. 4, no. 49, pp. 430-436, 2016.

[22] T. Mrowka, A Brief Introduction to Linear Analysis: Fredholm Operators. Geometry of Manifolds, Massachusetts Institute of Technology: MIT OpenCouseWare), Cambridge, MA, USA, 2004.

[23] A. A. Bakery and M. M. Mohammed, "Small pre-quasi Banach operator ideals of type orlicz- cesáro mean sequence spaces," Journal of Function Spaces, vol. 2019, Article ID 7265010, 9 pages, 2019.

[24] B. Altay and F. Başar, "Generalization of the sequence space $\ell(p)$ derived by weighted means," Journal of Mathematical Analysis and Applications, vol. 1, no. 330, pp. 147-185, 2007. 